\title{
Carumonam Sodium
}

National Cancer Institute

\section{Source}

National Cancer Institute. Carumonam Sodium. NCI Thesaurus. Code C79905.

The sodium salt form of carumonam, an $\mathrm{N}$-sulfo monocyclic beta-lactam antibiotic derived from sulfazecin. Carumonam has high resistance to beta-lactamases and activity against aerobic gram-negative bacteria. It reaches high urinary concentrations and may be used in the treatment of urinary tract infections. 